University of Wollongong

Research Online

Faculty of Engineering and Information

Faculty of Engineering and Information

Sciences - Papers: Part A

Sciences

2012

Design and development of compact readout electronics with silicon photomultiplier array for a compact imaging detector

Xiao-Hui Zhang

Graduate University of Chinese Academy of Sciences

Yu-Jin Qi

University of Wollongong, yujin@uow.edu.au

Cui-Lan Zhao

Graduate University of Chinese Academy of Sciences

Follow this and additional works at: https://ro.uow.edu.au/eispapers

Part of the Engineering Commons, and the Science and Technology Studies Commons

Research Online is the open access institutional repository for the University of Wollongong. For further information contact the UOW Library: research-pubs@uow.edu.au 


\title{
Design and development of compact readout electronics with silicon photomultiplier array for a compact imaging detector
}

\author{
Abstract \\ This work aims at developing compact readout electronics for a compact imaging detector module with \\ silicon photomultiplier (SPM) array. The detector module consists of a LYSO crystal array coupling with a \\ SensL's $4 \times 4$ SPM array. A compact multiplexed readout based on a discretized positioning circuit (DPC) \\ was developed to reduce the readout channels from 16 to 4 outputs. Different LYSO crystal arrays of $4 \times 4$, \\ $8 \times 8$ and $12 \times 12$ with pixel sizes of $3.2,1.6$ and $1.0 \mathrm{~mm}$ respectively, have been tested with the compact \\ readout board using a $137 \mathrm{Cs}$ source. The initial results show that the compact imaging detector module \\ with the compact multiplexed readout could clearly resolve $1 \mathrm{~mm} \times 1 \mathrm{~mm} \times 10 \mathrm{~mm}$ LYSO scintillation crystal \\ array except those at the edges. The detector's intrinsic spatial resolution up to $1 \mathrm{~mm}$ can be achieved \\ with the $3 \mathrm{~mm} \times 3 \mathrm{~mm}$ size SPMArray 4 through light sharing and compact multiplexed readout. Our results \\ indicate that this detector module is feasible for the development of high-resolution compact PET.

\section{Disciplines} \\ Engineering | Science and Technology Studies

\section{Publication Details} \\ Zhang, X., Qi, Y. \& Zhao, C. (2012). Design and development of compact readout electronics with silicon \\ photomultiplier array for a compact imaging detector. Chinese Physics C, 36 (10), 973-978.
}


Design and development of compact readout electronics with silicon photomultiplier array for a compact imaging detector

This article has been downloaded from IOPscience. Please scroll down to see the full text article.

2012 Chinese Phys. C 36973

(http://iopscience.iop.org/1674-1137/36/10/010)

View the table of contents for this issue, or go to the journal homepage for more

Download details:

IP Address: 130.130.37.84

The article was downloaded on 14/05/2013 at 02:17

Please note that terms and conditions apply. 


\title{
Design and development of compact readout electronics with silicon photomultiplier array for a compact imaging detector ${ }^{*}$
}

\author{
ZHANG Xiao-Hui(张晓慧 $)^{1,2}$ QI Yu-Jin(漆玉金) $)^{1 ; 1)}$ ZHAO Cui-Lan(赵翠兰) $)^{1,2}$ \\ ${ }^{1}$ Shanghai Institute of Applied Physics, Chinese Academy of Sciences, Shanghai 201800, China \\ ${ }^{2}$ Graduate University of Chinese Academy of Sciences, Beijing 100049, China
}

\begin{abstract}
This work aims at developing compact readout electronics for a compact imaging detector module with silicon photomultiplier (SPM) array. The detector module consists of a LYSO crystal array coupling with a SensL's $4 \times 4$ SPM array. A compact multiplexed readout based on a discretized positioning circuit (DPC) was developed to reduce the readout channels from 16 to 4 outputs. Different LYSO crystal arrays of $4 \times 4,8 \times 8$ and $12 \times 12$ with pixel sizes of 3.2, 1.6 and $1.0 \mathrm{~mm}$ respectively, have been tested with the compact readout board using a ${ }^{137} \mathrm{Cs}$ source. The initial results show that the compact imaging detector module with the compact multiplexed readout could clearly resolve $1 \mathrm{~mm} \times 1 \mathrm{~mm} \times 10 \mathrm{~mm}$ LYSO scintillation crystal array except those at the edges. The detector's intrinsic spatial resolution up to $1 \mathrm{~mm}$ can be achieved with the $3 \mathrm{~mm} \times 3 \mathrm{~mm}$ size SPMArray4 through light sharing and compact multiplexed readout. Our results indicate that this detector module is feasible for the development of high-resolution compact PET.
\end{abstract}

Key words: position readout electronics, silicon photomultiplier array, compact imaging detector

PACS: 87.85.Ng, 84.30.-r, 87.64.-t～DOI: $10.1088 / 1674-1137 / 36 / 10 / 010$

\section{Introduction}

Molecular imaging has been rapidly advancing toward multi-modality imaging. It requires fundamental improvements in instrumentation and imaging technique developments $[1,2]$. The multimodality imaging instrumentation heavily relies on novel detector technologies. The current research forefront of multimodality molecular imaging is to explore the integration of nuclear imaging (PET and SPECT) with MRI, which requires new imaging detector technologies to be immune to the magnetic field because the conventional nuclear imaging instrumentation based on the PMT is unsuitable in the magnetic field of MRI.

In recent years the silicon photomultiplier (SPM) has aroused considerable attention and interest for applications in nuclear molecular imaging, because of its appealing properties of great compactness with high gain $\left(\sim 10^{6}\right)$, fast response, low current consump- tion and insensitivity to the magnetic field [3-5]. The feasibility of the SPM used for the development of high-resolution compact PET and SPECT to integrate with MRI has been studied by many research groups, which is a useful tool for both functional and anatomic imaging $[6,7]$.

However, the design and development of highresolution detector modules based on SPMs into a practical imaging system are still great challenges. Optimizing the combination between scintillation crystals and SPMs, light sharing among the SPM array, and multiplexed readout schemes are crucial for the detector module to achieve a maximized performance.

In this work we use a LYSO scintillation crystal array coupled to a SensL's SPMArray4 for the development of a high-resolution detector module. A compact multiplexed readout based on a discretized positioning circuit has been developed to reduce the readout channels from 16 to 4 outputs. Different

\footnotetext{
Received 28 December 2011

* Supported by National Natural Science Foundation of China (10875162, 1105209)

1) Corresponding author's E-mail: yujinqi@yahoo.com

(C) 2012 Chinese Physical Society and the Institute of High Energy Physics of the Chinese Academy of Sciences and the Institute of Modern Physics of the Chinese Academy of Sciences and IOP Publishing Ltd
} 
light sharing between the crystal array and the SPM array was also studied. The performance of the detector module with the DPC readout was tested by a ${ }^{137}$ Cs source.

\section{Materials and methods}

\subsection{Detector module}

The detector module is composed of a LYSO crystal array and a SensL's SPMArray4. The LYSO crystal array was purchased from the Shanghai SICCAS High Technology Corporation, China. Three LYSO crystal arrays of $4 \times 4,8 \times 8$ and $12 \times 12$ crystal elements with pixel sizes of $3.2,1.6$ and $1.0 \mathrm{~mm}$ were tested with the SensL's SPMArray4. The crystal elements have a polish treatment on all sides. All other faces except for the light-out side are covered with a highly reflective PTFE-based material [8]. The SPMArray 4 is a $4 \times 4$ array of SPM pixels. Each pixel has an active area of $2.85 \mathrm{~mm} \times 2.85 \mathrm{~mm}$, made up of 3640 Geiger-mode avalanche photodiodes (micro- cells). The SPM array has a typical gain of $\sim 10^{6}$ at the manufacture-specified bias voltage of $29.5 \mathrm{~V}$, exceeding the breakdown voltage by $2.0 \mathrm{~V}$ [9].

The crystal array was coupled to the SPM array with a soft silicon pad with a thickness of $2 \mathrm{~mm}$ as a light guide to improve the light sharing among the SPM array elements. All the 16 channels of the SPM array were operated at the same bias voltage without tuning the individual bias for their gain variations.

\subsection{Readout electronics}

1) Design of the compact readout

The block diagram of the compact readout is shown in Fig. 1. The design scheme of the compact readout is based on the discretized positioning circuit (DPC) $[10,11]$ which can reduce the 16 readout channels of the SPMArray4 to 4 outputs. Then the 4 signals are amplified and shaped. A conventional center-of-gravity positioning algorithm was used to determine the position information of the incident gamma rays.

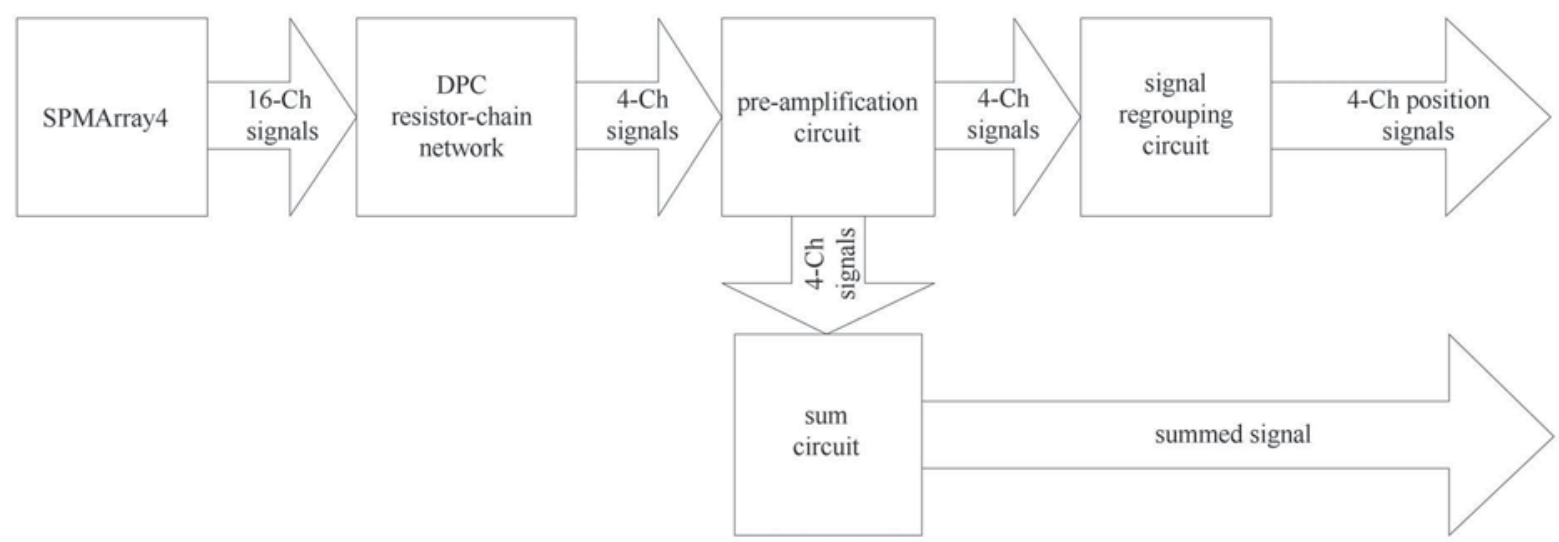

Fig. 1. Sketch diagram of the compact readout design for SPMArray4 silicon photomultiplier array.

2) The DPC resistor-chain network circuit

The DPC circuit consists of a $2 \mathrm{D}$ resistor-chain network to sum all the charges from the $4 \times 4$ SPM array and divides them into 4 outputs (A, B, C and D), as shown in Fig. 2. The main purpose of the circuit is to reduce the complexity of the readout and acquire electronic circuits. We have carefully tuned the resistor parameters to minimize the image shrinkage effect.

3) The pre-amplification circuit

The pre-amplification circuit is shown in Fig. 3. Since the detector module has a fast response to the incident gamma-rays which result in fast pulses with a rise-time of $\sim 5 \mathrm{~ns}$ and a decay time of $\sim 100 \mathrm{~ns}$ from the DPC circuit, we have to choose a low-noise, high-speed preamplifier with a broad bandwidth over $100 \mathrm{MHz}$ for the signal amplification. So the AD8066 amplifier chip from the ADI Inc. has been used in the preamplifier circuit. It is a rail-to-rail and high speed amplifier with $145 \mathrm{MHz}$ bandwidth, $180 \mathrm{~V} / \mu \mathrm{s}$ slew rate and a wide supply voltage range from $5 \mathrm{~V}$ to $24 \mathrm{~V}$ and the input resistance is $1000 \mathrm{G} \Omega$ to meet the demand of preamplifier in terms of high-speed, high input impedance and low noise.

Then the four outputs are amplified and shaped by low-noise charge-sensitive preamplifiers. Because the decay time of the LYSO crystal is about $40 \mathrm{~ns}$, we choose $R_{\mathrm{f}}=1 \mathrm{k} \Omega$ and $C_{\mathrm{f}}=4.7 \mathrm{pF}$ to provide stabil- 
ity of the transient response. C3 can reduce DC level noise. The amplification of the pre-amplification circuit is about 50, which can result in the amplitude of the output signals over 1 voltage.

4) The signal regrouping circuit

In order to meet the requirement of the center-ofgravity algorithm (shown in Eqs. $(1,2)$ ), we have to regroup the four (A, B, C and D) position signals into
$X^{+}, X^{-}, Y^{-}$and $Y^{+}$. Fig. 4 shows a regrouping circuit of A and B into $Y^{+}$, where the first stage of the circuit is to sum A and B and the second stage of the circuit is to reverse the negative signal to a positive signal to meet the requirement of our data acquisition system. Similarly, we have to sum B and D into $X^{+}$, $\mathrm{A}$ and $\mathrm{C}$ into $X^{-}$and $\mathrm{C}$ and D into $Y^{-}$.

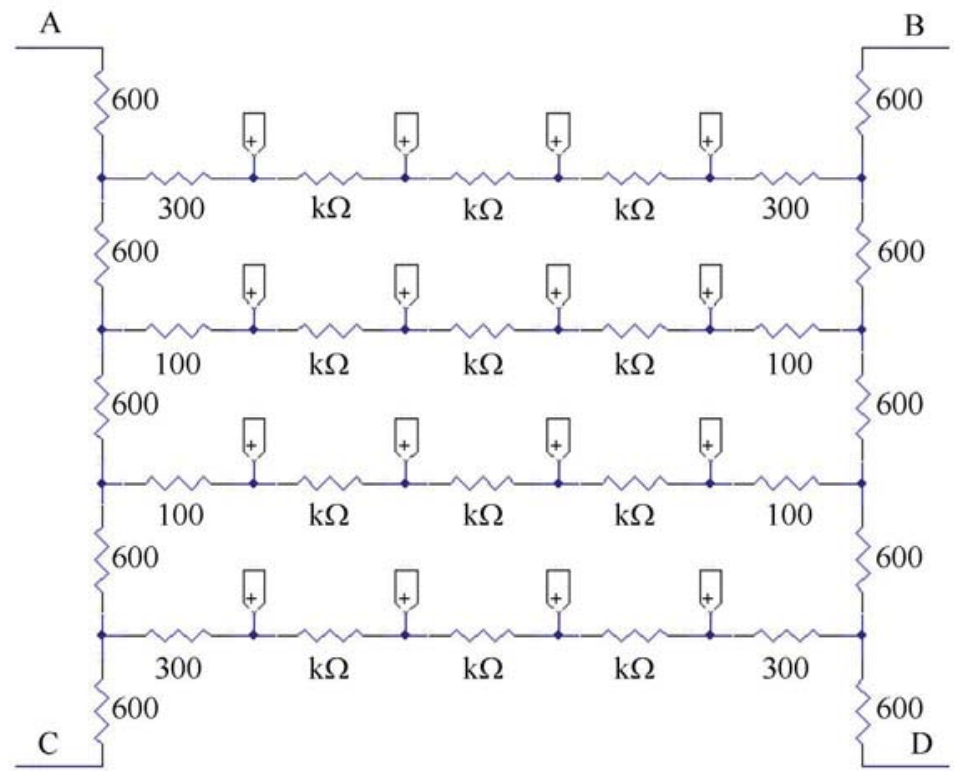

Fig. 2. The resistor-chain network of the readout circuit.

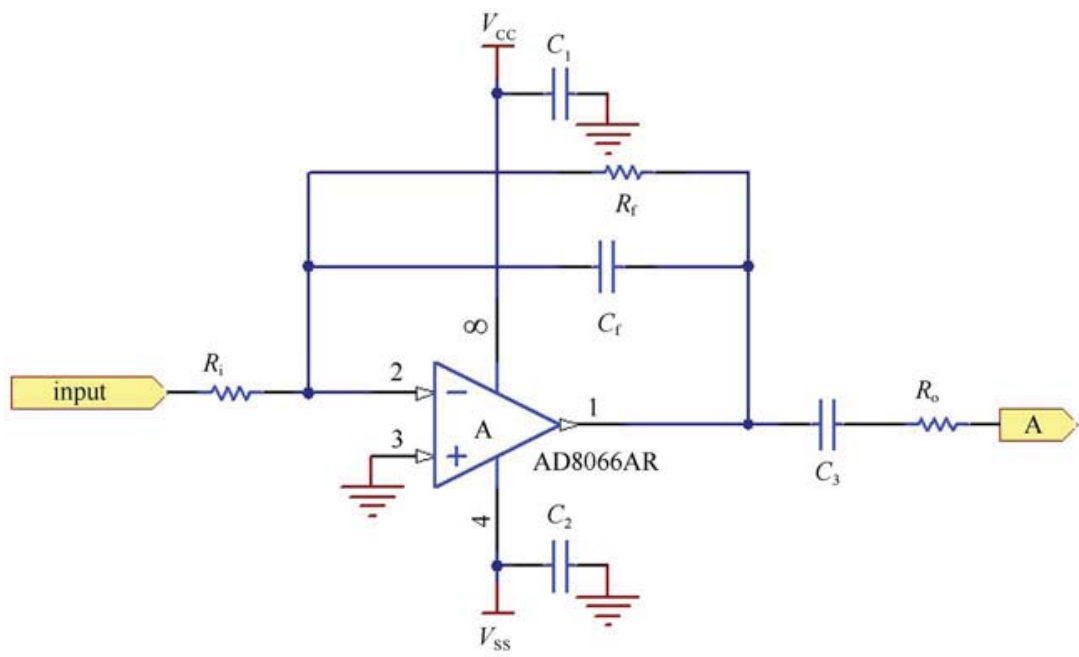

Fig. 3. The pre-amplification circuit. 


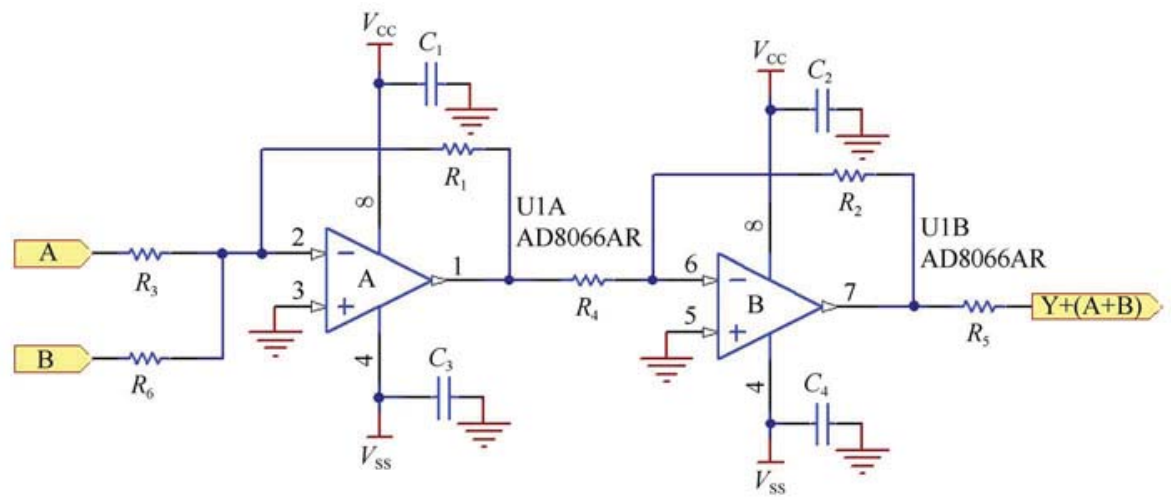

Fig. 4. The signal regrouping circuit.

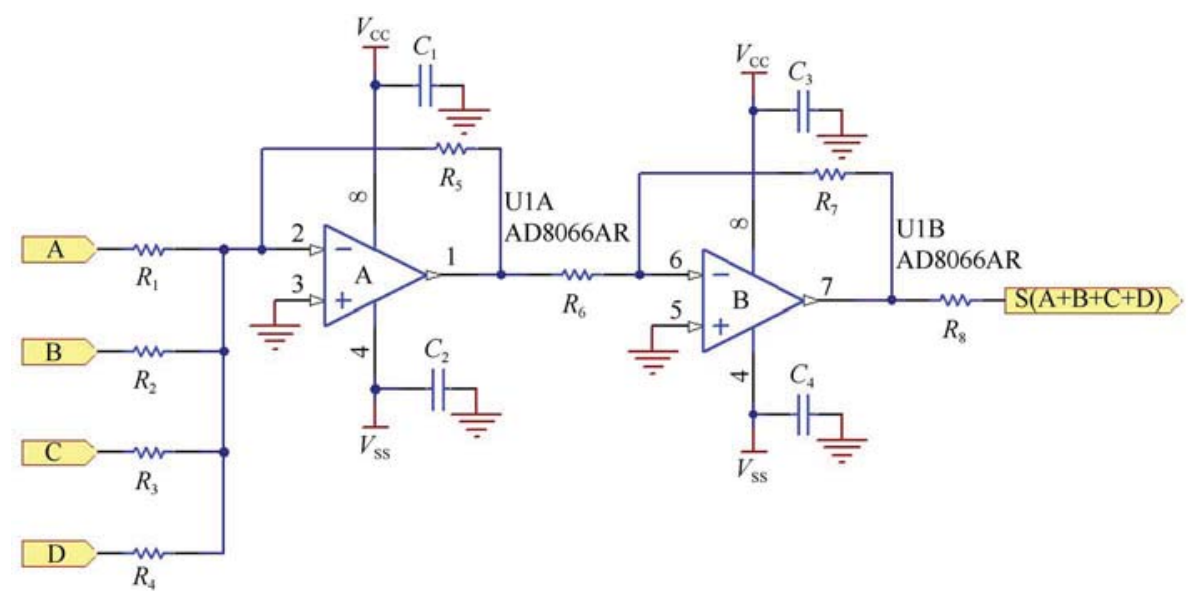

Fig. 5. The sum circuit.

5) The sum circuit

Figure 5 shows the sum signal circuit. It consists of a two-stage circuit. The first stage is to sum the four signals of A, B, C and D. The second stage is to reverse the sum signal from negative to positive. The sum signal is used as a trigger signal for the data acquisition system.

Then the four position signals $\left(X^{+}, X^{-}, Y^{+}\right.$and $Y^{-}$) are measured by a data acquisition (DAQ) system based on the NI PCI-6110 ADC card and the standard center-of-gravity method is used to determine the measured positions of the incident gamma rays. The event position and energy are calculated by the following equation:

$$
\begin{aligned}
& X=\frac{X^{+}-X^{-}}{X^{+}+X^{-}}, \\
& Y=\frac{Y^{+}-Y^{-}}{Y^{+}+Y^{-}}, \\
& E=X^{+}+X^{-}+Y^{+}+Y^{-} .
\end{aligned}
$$

\subsection{Detector module test}

A detector module with the compact readout board that we developed is shown in Fig. 6. The compact circuit board was implemented using SMD components in a two-layer PCB board. The performance of the detector module has been tested using a ${ }^{137} \mathrm{Cs}$ source with the gamma-ray energy of $662 \mathrm{keV}$. The bias voltage of the detector was set at $29.5 \mathrm{~V}$.

The measured position signal with the trigger signal in an oscilloscope is shown in Fig. 7. The typical position signal of the detector module with our readout board has an amplitude of $\sim 2 \mathrm{~V}$ and a rise time of $\sim 60$ ns with the ${ }^{137} \mathrm{Cs}$ source.

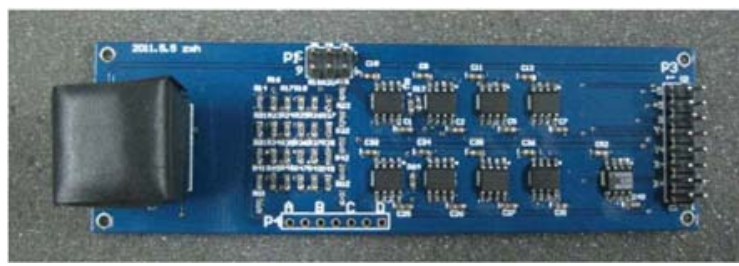

Fig. 6. Photography of the detector module with the compact readout and amplification board. 


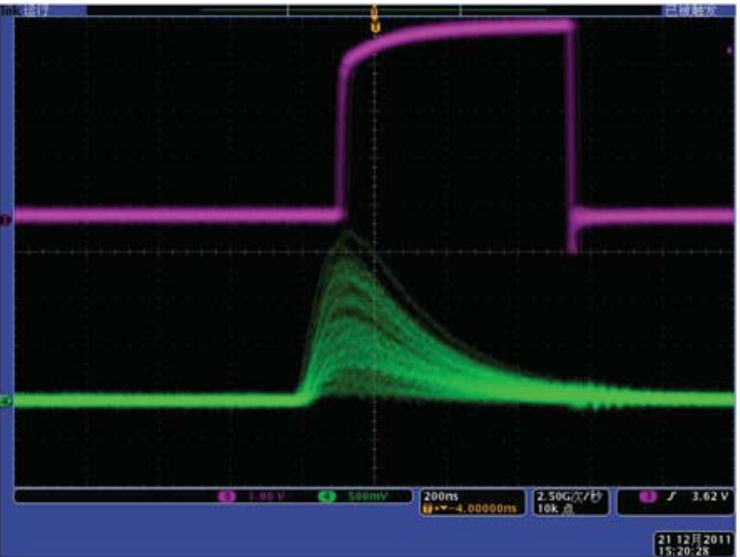

Fig. 7. The waveforms captured from the oscilloscope. Top: the trigger signal; Bottom: a typical position signal.

\section{Results}

Figure 8 shows the measured raw flood images of the detector module's responses using ${ }^{137} \mathrm{Cs}$ source with $4 \times 4$ and $8 \times 8$ LYSO crystal arrays, respectively. For the $4 \times 4$ crystal array with a pixel size of $3.2 \mathrm{~mm}$, all the crystal elements are well resolved. For the $8 \times 8$ crystal array with a pixel size of $1.6 \mathrm{~mm}, 7 \times 7$ crystal
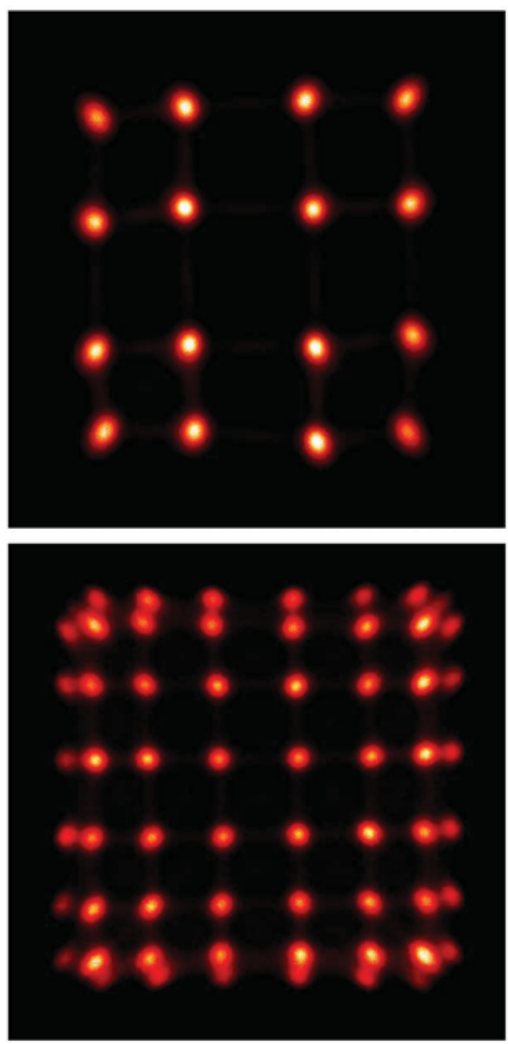

Fig. 8. The measured raw flood images of the detector module with two LYSO crystal arrays using ${ }^{137} \mathrm{Cs}$ source. Top: with a $4 \times 4$ crystal array; Bottom: with a $8 \times 8$ crystal array. elements are well resolved. However, the separation for those crystal elements in the edges of the detector is marginal because of the distortion. Both flood images show a fairly good uniformity on the detector's response.

Figure 9 shows the measured raw flood image of the detector module with $12 \times 12$ crystal array with a pixel size of $1.0 \mathrm{~mm}$. Only $10 \times 10$ crystal elements are clearly identified except those in the edge region of the detector. The overall spatial response of the detector module remains a good shape as compared with that of the detector module with $4 \times 4$ and $8 \times 8$ crystal arrays, which indicates that the performance of our readout board is consistently good and reliable with different sizes of LYSO crystal arrays.

Then we choose two regions-of-interest (ROIs) of
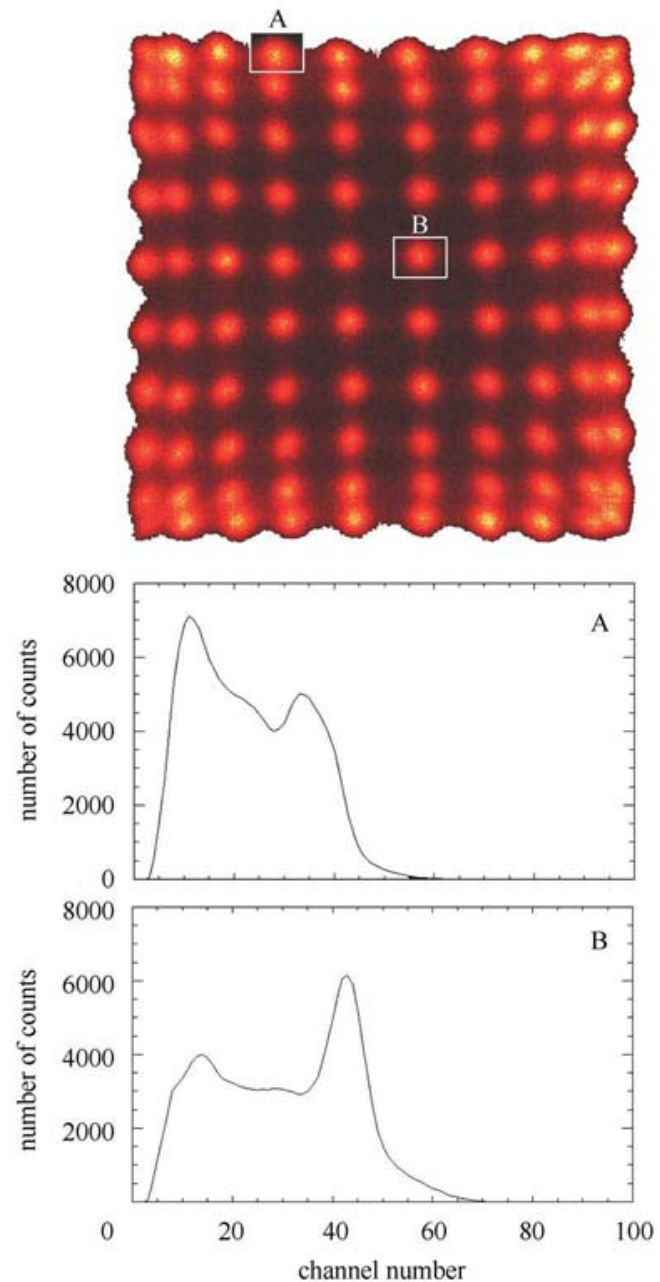

Fig. 9. The measured responses of the detector module with a $12 \times 12$ LYSO crystal array using ${ }^{137} \mathrm{Cs}$ source. Top: the measured raw flood image; Middle: the measured energy spectrum from a crystal element in the edge of the detector; Bottom: the measured energy spectrum from a crystal element in the center region of the detector. 
$\mathrm{A}$ and $\mathrm{B}$ around individual crystal elements seen in the raw flood image to measure the energy spectrum with the ${ }^{137} \mathrm{Cs}$ source. The measured energy spectra are shown in Fig. 9. As expected, ROI-A in the edge of the detector has a worse energy resolution of $\sim 29.4 \%$ compared with ROI-B in the central region of the detector which has a good energy resolution of $\sim 16.3 \%$.

From the measured flood image and the energy spectra, we can see that the detector's response in the central region has a better performance than that in the edges because the SPM array in the central region has a better collection and sampling of the scintillation light photons from the crystal array than those in the edges.

\section{Conclusion and discussion}

We have successfully developed a compact detec-

\section{References}

1 Weissleder R, Ross B D, Rehemtulla A, Gambhir SS. Molecular Imaging: Principles and Practice, PMPH-USA Press, Aug. 2010

2 Judenhofer M S, Wehrl H F, Newport D F et al. Nat. Med., 2008, 14(4): 459-465

3 Buzhan P et al. Nucl. Instrum. Methods A, 2003, 504

4 Jackson J C, Hughes P J, Herbert D, Stewart A, Wall L. IEEE Lasers \& Electro-Optics Society, 2006, 723-724

5 WANG Y. Nuclear Electronics \& Detection Technology, 2006, 26(3): 280-283 (in Chinese)

6 Grazioso R, ZHANG N, Corbeil J et al. Nucl. Instrum. tor module based on a LYSO crystal array coupled to a SensL's $4 \times 4$ SPM array (SPMArray4). A compact multiplexed readout board based on the DPC readout scheme was developed for this detector module. The performance of the detector module with the compact readout electronics has been evaluated with different LYSO crystal arrays. Our study has shown that the detector module can provide good performance in both spatial and energy resolution. Up to $1 \mathrm{~mm}$ intrinsic spatial resolution can be achieved with the $3 \mathrm{~mm} \times 3 \mathrm{~mm}$ cell-size SPM array using conventional light sharing and charge multiplexed readout method. The results show that the conventional light sharing and charge multiplexed readout method is suitable for the high-performance imaging detector module. This detector module and readout electronics are feasible for the development of high-resolution compact micro-PET.

Methods A, 2006, 569: 301-305

7 Jung J H, Choi Y. MR Compatible Brain PET Using Tileable GAPD Arrays. IEEE Nuclear Science Symposium Conference Record, M13-27

8 Datasheet: SICCAS LYSO. http://www.siccas.com/ index_cn.asp

9 Datasheet: SensL SPMArray4. http://sensl.com

10 Siegel S, Cherry S R, Shao Y. IEEE Trans. Nucl. Sci., 1996, 43(3): 1634

11 Cherry S R, SHAO Y, Siegel S, Silverman R W, Mumcuoglu E, Mesdors, Phelps M E. IEEE Trans. Nucl. Sci., 1996, 43(3): 1932-1937 